\title{
Analytic Theory of Two Wave Interactions in a waveguide with a $\chi^{(3)}$ nonlinearity.
}

\author{
Neil G. R. Broderick ${ }^{1}$, Max A. Lohe ${ }^{2}$, Timothy Lee ${ }^{3}$ and Shaaram \\ Afshar V. ${ }^{2}$ \\ 1) Department of Physics, University of Auckland, Auckland, NZ \\ 2) Institute for Photonics \& Advanced Sensing (IPAS), The University of Adelaide, 5005, \\ Australia \\ 3) Optoelectronics Research Centre, University of Southampton, Southampton SO17 1BJ, UK \\ n.broderick@auckland.ac.nz
}

In a material with a Kerr nonlinearity such as glass it is possible to generate the third harmonic of an incident wave, however in general, due to material dispersion such a process is not phase-matched and so rarely occurs with any useful efficiency. The theory of third harmonic generation thus remained a theoretical curiousity which meant that the work of Armstrong et al. [1] who found an analytic solution to the problem (which was further extended by Puell and Vidal [2]) has been forgotten resulting in later authors having to re-derive similar solutions [3]. The later work was influenced by the fact that efficient third harmonic generation is possible in waveguides using modal dispersion to ensure phase matching between light at frequency $\omega$ in the fundamental mode and light at frequency $3 \omega$ in a higher order mode. Such phase matching is only possible in nanowires where the diameter of the waveguide is less than the wavelength of light and typically the refractive index contrast is large since the cladding is usually assumed to be air. Futhermore since the early work of Armstrong et al. the availability of high power lasers at different wavelengths has greatly increased and so it is possible to consider the nonlinear interaction of two strong pump beams coupled by a third order nonlinearity and there is thus a need to re-examine the problem looking more generally at the different types of solutions. Assuming that the interactions take place within an optical waveguide leads to the following set of coupled mode equations[3]:

$$
\begin{aligned}
& \frac{\partial A_{1}}{\partial z}=i n^{(2)} k_{1}\left[\left(J_{1}\left|A_{1}\right|^{2}+2 J_{2}\left|A_{3}\right|^{2}\right) A_{1}+J_{3}\left(A_{1}^{*}\right)^{2} A_{3} e^{i \delta \beta z}\right] \\
& \frac{\partial A_{3}}{\partial z}=i n^{(2)} k_{1}\left[\left(6 J_{2}\left|A_{1}\right|^{2}+3 J_{5}\left|A_{3}\right|^{2}\right) A_{3}+J_{3}^{*} A_{1}^{3} e^{-i \delta \beta z}\right]
\end{aligned}
$$

where the various $J_{i}$ coefficients are related to the modal overlaps between the fundamental and third harmonic fields [3]. In Eqs. (1) $A_{1}$ is the slowly varying amplitude of the fundamental field at frequency $\omega$ while $A_{3}$ is the envelope of the third harmonic field at frequency $3 \omega$. The Kerr nonlinearity is given by $n^{(2)}$ while $k_{1}=\omega / c$. The phase matching is determined by $\delta \beta=\beta(3 \omega)-\beta(\omega)$. Even though we have assumed that the interaction occurs in an optical waveguide the equations are formally identical to those considered by Armstrong $e t$ al. for plane wave interactions with only the relative ratios of the coefficients being different. Indeed this is one of the main attractions of doing these experiments in a waveguide geometry, since by careful design of the waveguides it is possible both to engineer a small value of the phase mismatch $\delta \beta$ but also to control the relative sizes of the different modal overlaps. Note that it is also possible to define the modes so that $J_{3}$ is always purely real and so we will assume that this is the case throughout this paper.

The definition of the fibre modes used to derive Eqs. 1 implies that the power in each mode is given $\left|A_{i}\right|^{2}$ and so conservation of energy immediately implies that $\left|A_{1}\right|^{2}+\left|A_{3}\right|^{2}$ is constant [which can be also proved easily from Eqs. (1)]. This allows us to introduce the real variables $v, \phi_{1}$ and $\phi_{3}$ through:

$$
A_{1}=P_{0} v(z) e^{i \phi_{1}(z)}, \quad A_{3}=P_{0}(1-v) e^{i \phi_{3}(z)}
$$

and define a new variable $\theta(z)=\phi_{3}(z)-3 \phi_{1}(z)+\delta \beta z$. Then Eqs. (1) imply

$$
\begin{aligned}
& \frac{d v}{d \tau}=-2 v \sqrt{v(1-v)} \sin \theta \\
& \frac{d \theta}{d \tau}=a+2 b v+(4 v-3) \sqrt{\frac{v}{1-v}} \cos \theta
\end{aligned}
$$

where

$$
\begin{aligned}
& \tau=n^{(2)} k_{1} J_{3} P_{0} z \\
& a=\frac{\delta \beta}{n^{(2)} k_{1} J_{3} P_{0}}+\frac{3\left(-2 J_{2}+J_{5}\right)}{J_{3}} \\
& b=-\frac{3\left(J_{1}-4 J_{2}+J_{5}\right)}{2 J_{3}}
\end{aligned}
$$


and $P_{0}$ is the total power in the system and $\tau$ can be considered a normalised distance. Starting from these equations other authors have looked at how the third harmonic grows from noise and have found analytic solutions for this. However we are interested in the more general case in which both the fundamental and 3rd harmonic beams are initially non-zero. This general case has not previously been examined as far as we know. It is also worth noting that introducing the variables $v, \phi_{1}$ and $\phi_{2}$ leads to some unphysical solutions as well as eliminating the physical solution of $A_{1}(z)=0, A_{3}(z) \neq 0$. However as long as we are aware of this it does not cause significant problems and such solutions can be eliminated (or found) using a different parameterization.

In order to solve Eqs. (3) we start by noticing that they can be derived from a Hamiltonian

$$
H=-a v-b v^{2}+2 v \sqrt{v(1-v)} \cos \theta
$$

which is independent of $\tau$ and so is a conserved quantity[2,4]. For any particular trajectory starting from $\left(v_{0}, \theta_{0}\right)$ there is a corresponding value of $H\left(v_{0}, \theta_{0}\right)=H_{0}$ and so we can use Eq. (5) to solve for $\theta(\tau)$ in terms of $v$ and $H_{0}$. This leaves one equation for $v$ which can be rewritten as

$$
\dot{v}^{2}=4 v^{3}(1-v)-\left(H_{0}+a v+b v^{2}\right)^{2}=Q(v)
$$

where $Q(v)$ is a quartic polynomial. The standard solutions to such an equation are given in terms of elliptic functions[5]. Also note that $H_{0}=0$ corresponds to the case where there is no initial third harmonic and the explicit solutions in this regime where given by Armstrong et al. [1]. These analytic solutions are useful since they show how the final state of the system depend on the parameters of the problem such as the initial power $P_{0}$. They also provide a useful check on numerical solutions to ensure that what is observed is real rather than a numerical artifact (see for example the rapid phase evolution in Fig.2a). However in many cases sufficient insight can be found by looking a contour plot of $H$ for different values of $a$ and $b$. Here each contour corresponds to a different trajectory and it is easy to see the fixed points and whether or not they are stable. Three typical contour plots are shown in Fig. 1.
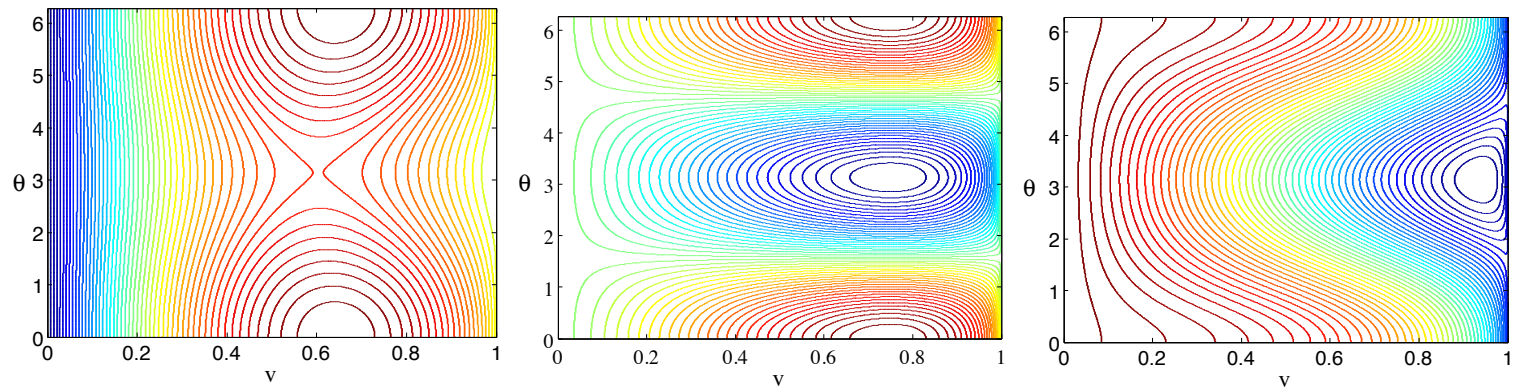

Fig. 1. Contour plots of the Hamiltonian for three different cases. In the first $a=-20$ and $b=16$. In the second $a=b=0$ and in the third $a=b=1$.

\section{Stationary Solutions}

In order to understand the general behaviour of the solutions it is useful to first characterise the fixed points. Eqs. (3) permit three types of stationary solutions. The first is when $v=0$, the second is when $v=1$ and the last is when $\sin \theta=0$. The fixed point at $v=1$ is unphysical since the original equations do not have a similar fixed point while the fixed point at $v=0$ is physical but it is uninteresting as physically it represents all of the light being in the third harmonic and while this is a valid solution it is also an unstable one. It is worth nothing that an immediate consequent of $v=0$ and $v=1$ being fixed points is that complete harmonic conversion efficiency can never be achieved since that would require a trajectory starting from one fixed point and ending on another. The case when $\sin \theta=0$ is more interesting since that implies that $\cos \theta= \pm 1$ and so requiring that $\dot{\theta}=0$ is equivalent to solving a cubic equation for $v$. Since a cubic equation always has at least one real root we can show that there is always one fixed point for the system (i.e. there is always a real root to the cubic between 0 and 1). There can also be 3 fixed points as in Fig. 1(a),(b) and numerically such cases appear to have more interesting dynamics than the case of a single fixed point. We have also analysed the conditions for the stability of the fixed points and these results will be presented at the conference.

\section{Seeded Third Harmonic Generation}

Previously most authors have concentrated on the problem of efficient 3rd harmonic generation and have shown that maximum conversion efficiency occurs when $a=b=0$. The possible trajectories here are shown in Fig. 1(b), (nothing that $v=1$ corresponds to all the power being in the fundamental) which however does not show anything about the fibre length required to obtain maximum 3rd harmonic. Instead we solve the original ODE's for a variety of cases, the first when $v(0)=0.999$ and $a=b=0$. This result is shown in Fig. 2(a) and shows maximum 
conversion efficiency. In contrast in Fig. 2(b) and (c) we set $a=7$ and $b=-6.8$ corresponding to realistic values for a soft-glass fibre taper. In both cases $v=0.9$ and we vary theta from $\pi$ in Fig. 2(b) to $\pi+2.5$ in Fig. 2(c). It is clear that changing the initial phase has a dramatic effect on the amount of third harmonic generated (from nearly $80 \%$ to less than $13 \%$ ) while the length required for maximum conversion efficiency also changes significantly. While the effect of the initial phase is not surprising for a parametric process it shows that with careful control seeded harmonic generation can result in useful conversion efficiencies.
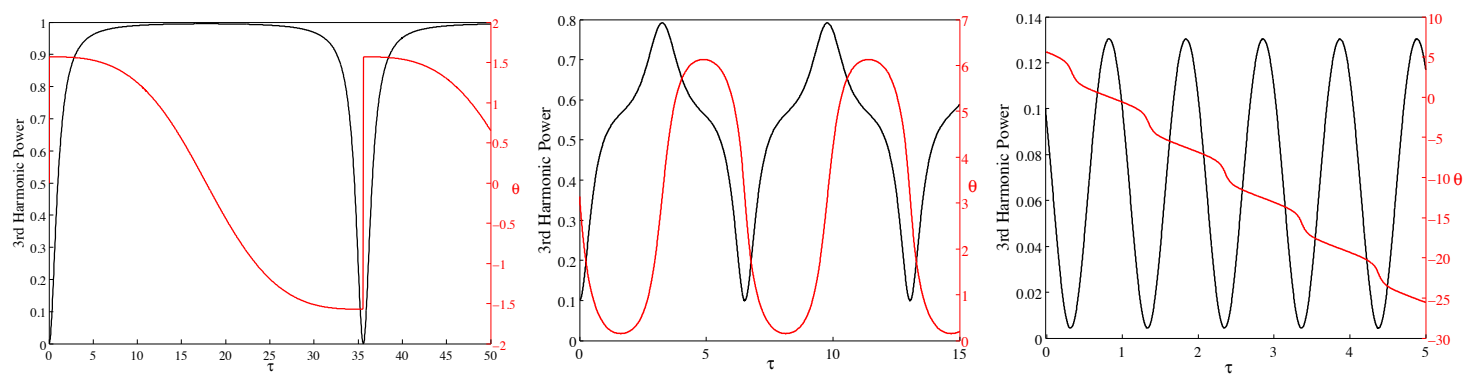

Fig. 2. (a) Growth of the 3rd harmonic for $v(0)=0.99999$ and $a=b=0$. (b) Growth of the 3rd harmonic for $v(0)=0.9, \theta(0)=\pi$ and (c) $\theta=\pi+2.5$. In (b) and (c) $a=7$ and $b=-6.8$ corresponding to realistic parameters for a soft glass fibre taper.

Conclusions and Discussion In this work we have extended the analysis of Armstrong et al. on third harmonic generation to look at more general interactions between two co-propagating beams at the fundamental and third harmonic in a $\chi^{(3)}$ nonlinear waveguide. We have found cases of stable and bistable behaviour along with interesting phase effects. Importantly the parameters determining the possible solutions depend on the mode overlaps which for an optical nanowire can be engineered to have a wide range of values. As efficient third harmonic in optical nanowires has already been observed it the more interesting dynamics presented in this paper should be capable of being observed and utilised in the near future.

Practically we note that in most experiments the wavelength of the source can usually be tuned somewhat allowing considerable variations in $\delta \beta$ and so we can always set $a$ to any required value. The parameter $b$ is fixed by the parameters of the taper and so cannot be varied during the course of an experiment. However there is scope for altering $b$ by choosing which modes are phase-matched and also the precise taper diameter.

Physically in deriving the original PDE's a number of approximations have been made. Firstly we have assumed that no Raman nonlinearity is present which again is something that can be avoided since the power required here are low since the interaction is nearly phase matched. Secondly we have neglected the effect of spontaneous parametric down-conversion. Such an effect would be small but would for example be dominant if we only launched the third harmonic into the fibre when it would need to be included as an additional noise term. Since any parametric fluorecsence light would grow from vacuum noise the length scales involved would be considerablely longer than than any practical optical nanowire ( $\mathrm{kms}$ as opposed to $\mathrm{mm}$ ).

\section{References}

[1] J. A. Armstrong et al. "Interactions between Light Waves in a Nonlinear Dielectric", Phys. Rev. 127(6) pp 1918 (1962). [2] H. Puell and C. Vidal "Optimum conditions for nonresonant third harmonic generation", IEEE J. Quant. Elect. 14(5) pp 364 (1978)

[3] V. Grubsky and A. Savchenko, "Glass micro-fibers for efficient third harmonic generation", Opt. Exp. 13(18) pp 6798 (2005). [4] H. Steffensen, et al. , "Full and semi-analytic analyses of two-pump parametric amplification with pump depletion", Opt. Exp. 19(7) pp 6648 (2011). [5] I. S. Gradshteyn and I. M. Ryzhik, "Table of Integrals, Series and Products" (Academic, 1965) 